Nadia Rosenthal is an example of the reverse brain drain. Her move, just under a year ago, from Harvard Medical School to the European Molecular Biology Laboratory (EMBL) in Monterotondo was triggered by the transfer in the opposite direction of Klaus Rajewsky. She now has his old job running EMBL's mouse research program, and he has moved to Harvard (Nature Med. 7, 392; 2001). Rosenthal shared her views of the two research settings with Nature Medicine.

\title{
Nadia Rosenthal
}

Known predominantly for her research into heart development in the United States, one of Nadia Rosenthal's current areas of investigation at EMBL is the manipulation of growth factors produced by skeletal muscle to improve regenerative capacity. Having been involved in cloning the insulin gene years ago, Rosenthal was mindful of its cousin, insulin growth factor-1 (IGF-1), which is produced in muscle. "I discovered that we could attenuate or reverse many of the degenerative processes of muscle by supplementing a particular form of IGF-1."

The newest, as yet unpublished, data is based on the observation that much of muscle's regenerative potential depends on its ability to recruit new cells. Her team has engineered IGF-1 genes into skeletal and cardiac muscle in the context of a disease state such as muscular dystrophy and myocardial infarct. The presence of additional growth factor bestows regenerative properties: tissue does not scar as frequently and animals do not show standard signs of aging. "We're unraveling the mechanism by which IGF-1 recruits stem cells and we're working on ways to induce the endogenous gene so we can change the way in which muscle responds to injury and disease, and does a better job of renewing itself."

But it's still early days for the EMBL team. Rosenthal runs a section of 60 scientists, including six faculty members, most of whom only arrived six months ago. She herself is approaching her one-year anniversary running the department.

In talking to Rosenthal, the overriding impression is that the sense of a scientific community is what drew her from Harvard to Monterotondo, just outside Rome-the richness of it at EMBL and the lack of it at Harvard. But there were also more tangible considerations associated with the move. "The idea of having strong core facilities and hard money so you don't have to spend all your time writing grants really appealed to me," she says. "Plus, I was drawn to the organizational structure: an international group of young, motivated people, fully funded for a decade, who didn't suffer what I had seen my colleagues go through in the States, which is being drowned in the effort to get a lab up and running. Without those pressures, scientists can explore more radical ideas, and I was attracted to that."

Despite the praise, Rosenthal initially identified a gap in the EMBL faculty-it lacked a strong emphasis on molecular medicine. But this was precisely the hole that EMBL director general Fotis Kafatos wanted to plug, and Rosenthal has taken on the task. "I was intrigued with the possibility of grouping together researchers [who] would have no particular discipline except the model of a mouse as a focus. Because everyone here is working on the mouse, it speeds things up tremendously."

Rosenthal finds the tenure system in the big American universities such as Harvard relatively "demoralizing," since so few researchers actually achieve the status. Researchers leave EMBL after ten years. "Not because they're kicked out," she explains, but because they are given a decade to work in the organization. "This means that nobody is fighting to get through the eye of the needle into a tenured position, so that whole sense of trickle-down anxiety that is rife at Harvard doesn't exist at EMBL. I don't mean to sound evangelistic, but this really is an extraordinary institution."

She retains, however, the "deepest respect, affection and nostalgia" for research in the United States. Without the financial backing that is available there through the National Institutes of Health, European science struggles. "The funding is subject to the same sorts of ebbs and flows as the national economy of that country," Rosenthal observes. She finds it "embarrassing" how little the Italian government puts into research, and says it would be "folly" to suggest that Europe is a better place to do science than the United States, although she adds, “But I didn't really move from America to Europe, I moved from America to the EMBL."

Within the last few months, she has been involved in the Sixth Framework Programme. This is the European Union's funding system for research, and will distribute a total budget of $€ 17.5$ billion ( $\$ 17$ billion) over the next four years. Rosenthal has found the mechanism irkingly different from the US procedure. "The EU has a number of initiatives that they're trying out on the scientific community that are interesting and innovative but risky."

Unlike their NIH counterparts, EU grants are never small or individual; they cover large, integrated labs, a strategy that is intended to stimulate cooperation among the European scientific community and blur geographical boundaries. "The problem," says Rosenthal, is that rather than announce areas of interest, "the EU decided to have the scientific community come up with suggestions as to what they should fund. They got 15,000 suggestions when they were expecting a few hundred!"

She joined a life-sciences review team brought in to look at 100 suggestions at a time. Eventually a few ideas were selected. The problem now is that researchers are hastily trying to configure themselves into groups that can bid in the selected grant areas. "For instance, one of the priorities is cardiovascular research. Now I have to find out who I can join. I make lots of phone calls. It's who you know and word of mouth."

It's "regrettable", she says, that the European Union has decided to "completely ignore the good working grant system established in America." But the "good news" comes back to that sense of community that she finds so important to her work: "I'm getting to know a lot of European colleagues. Collaboration is the name of the game here and it's not the case in America."

Karen Birmingham, London 